Moroccan J. of Pure and Appl. Anal. (MJPAA)

Volume 8(1), 2022, Pages 54-66

ISSN: Online 2351-8227 - Print 2605-6364

DOI: $10.2478 / \mathrm{mjpaa}-2022-0005$

\title{
On some results on interpolative Kannan-type and CRR-type contractions.
}

\author{
Amnay EL AMRI ${ }^{1}$, Youssef EL FOUTAYENI ${ }^{2}$, LATe El Miloudi MARHRANI ${ }^{3}$
}

\begin{abstract}
Aвstract. Recently, Karapinar, Aydi and De Hierro introduced the notions of interpolative Kannan-type contractions and interpolative CRR-type contractions and proved that they possess a fixed point when the space is complete. In this paper, we show that the existence of fixed points for interpolative Kannan-type and CRR-type contractions remains true for a metric space which is not necessarily complete and we give a more precise result concerning the behaviour of Picard sequences of arbitrary initial point. We also study some particular classes of interpolative Kannan-type and CRR-type contractions.
\end{abstract}

Mathematics Subject Classification (2020). 46T99; 47H10; 54H25

Key words and phrases : fixed-point, Kannan-type contractions, CRR-type contractions.

In memory of my thesis co-supervisor, Pr. El Miloudi MARHRANI

who passed away during the preparation of this work.

\section{Introduction and main results}

Recently, Karapinar, Aydi, and De Hierro considered two classes of maps in [4] and [2] (see the case where $\psi=\lambda t$ page 5, Corollary 3) : the interpolative Kannan-type contractions and the interpolative CRR-type contractions. We recall the definitions of these classe of maps :

Received February 08, 2021 - Accepted May 12, 2021.

(C) The Author(s) 2021. This article is published with open access by Sidi Mohamed Ben Abdallah University.

${ }^{1}$ Hassan II University, FSBM: amnayelamri95@gmail.com (Corresponding Author)

${ }^{2}$ Hassan II University, FSBM : foutayeni@gmail.com

${ }^{3}$ Hassan II University, FSBM . 
Definition 1.1. Let $(X, d)$ be a metric space, and $T: X \longrightarrow X$.

We denote by Fix $(T)$ the set of fixed points of $T:\{x \in X, T(x)=x\}$.

$T$ is said to be an interpolative Kannan-type contraction if and only if : $\exists \alpha \in] 0,1[, \forall x, y \notin F i x(T)$,

$$
d(T x, T y) \leq \lambda d(x, T x)^{\alpha} d(y, T y)^{1-\alpha}
$$

for some $\lambda \in[0,1[$.

And $T$ is said to be an interpolative CRR-type contraction if and only if :

$$
\begin{gathered}
\exists \alpha, \beta, \gamma \in] 0,1[, \alpha+\beta+\gamma=1, \forall x, y \in X \backslash \operatorname{Fix}(T), \\
d(T x, T y) \leq \lambda d(x, y)^{\alpha} d(x, T x)^{\beta} d(y, T y)^{\gamma}
\end{gathered}
$$

for some $\lambda \in[0,1[$.

The authors prove that when the space is complete these maps always have a fixed point. The fixed-point theorem for interpolative Kannan-type contractions is proved in [4], and the theorem concerning interpolative CRR-type contractions is proved as a corollary of a more general theorem in [2] (page 5, corollary 3). In addition to the existence of a fixed point, some information on the behaviour of Picard sequences for arbitrary initial point is given. Namely they proved the following two theorems:

\section{Theorem 1.1.}

Let $X$ be a complete metric space and $T: X \rightarrow X$ an interpolative Kannan-type contraction. Then, for all $x_{0}$ in $X$, the sequence $\left(T^{n}\left(x_{0}\right)\right)_{n}$ converges to a fixed point of $T$.

Theorem 1.2. Let $X$ be a complete metric space and $T: X \rightarrow X$ an interpolative CRR-type contraction. Then, for all $x_{0}$ in $X$, the Picard sequence of initial point $x_{0}$ converges to a fixed point of $T$.

In the second section, we strengthen the fixed-point theorems that have been proved in previous articles. In fact we shall assume less assumptions and obtain more informations in the conclusions. Firstly we will establish these two results:

Theorem 1.3. Let $X$ be a metric space and $T: X \rightarrow X$ an interpolative Kannan-type contraction. Then, $T$ has a fixed point.

Theorem 1.4. Let $X$ be a metric space and $T: X \rightarrow X$ an interpolative CRR-type contraction. Then, $T$ has a fixed point.

Secondly, we will focus on the behaviour of Picard sequences for an arbitrary initial point and prove that they are stationary and their limit is a fixed point. In the third section, we introduce the notion of finite interpolative Kanan-type contraction and finite interpolative CRR-type contraction and show that these notions are related to the boundlesness of the metric space. In the fourth and last section, we study the space of continuous interpolative Kannan-type or CRR-type contractions in the special case of a connected metric space. This assumption on the space allows us to determine all the continuous interpolative Kannan-type and CRR-type contractions defined on it.

Except for the second section, we chose in this article to expose only the proofs concerning interpolative CRR-type contractions. The reason of this choice is that all the proofs and constructions are very similar and may be a little more complicated in the case of interpolative 
CRR-type contractions. The reader may easily adapt these proofs to the case of interpolative Kannan-type contractions.

\section{Extension of fixed-point theorems for Interpolative Kannan-type and CRR-type contrac- tions}

Our purpose in this section is to extend the theorems 1.1 and 1.2. In order to prove these two theorems, Karapinar, Aydi and Roldan Lopez De Hierro $([4,2])$ followed these steps. The first step is to consider, for an arbitrary $x_{0}$ in $X$, the Picard sequence of initial point $x_{0}$ i.e. $\left(T^{n}\left(x_{0}\right)\right)_{n}$. The second step is to prove that the sequence considered is Cauchy using the contractive assumption on the map. The third and last step is to deduce that the sequence is convergent using the completeness of the space and prove that the limit is a fixed point of the map.

In this part, we shall prove, using a different method, stronger results. In the subsection 2.1 we prove the existence of a fixed point for interpolative Kannan-type and CRR-type contractions which are defined on non-complete metric spaces.

In the subsection 2.2 we study the behaviour of Picard sequences for interpolative Kannan-type and CRR-type contractions. This study allows us to improve the results of subsection 2.1. The final theorem is stated as follows :

Theorem 2.1.

Let $X$ be a metric space and $T: X \rightarrow X$ an interpolative Kannan-type or CRR-type contraction. Then, for all $x_{0}$ in $X$, the sequence $\left(T^{n}\left(x_{0}\right)\right)_{n}$ is stationary and the limit is a fixed point of $T$.

The reader may notice that in this theorem we assume weaker hypothesis than in theorems 1.1 and 1.2 and that we obtain stronger results. Indeed, the completeness of the space $X$ is removed and the theorem gives more informations about the behaviour of Picard sequences.

\subsection{Existence of a fixed point.}

First, we state the following proposition for interpolative Kannan-type contractions:

Proposition 2.2. Let $T$ be an Interpolative Kannan-type contraction on a metric space $(X, d)$. If $\inf _{x \backslash F i x(T)} d(x, T x)=0$, then $T$ is constant on $X \backslash F i x(T)$.

Proof. Let $x \in X \backslash F i x(T)$. According to the hypothesis :

$$
\exists\left(x_{n}\right)_{n \in \mathbb{N}} \subset X \backslash \operatorname{Fix}(T), \lim _{n \rightarrow \infty} d\left(x_{n}, T x_{n}\right)=0,
$$

In fact, it is clear that the lower bound defined on the hypothesis cannot be reached. We use the inequality (1.1) of Kannan-type contractions for $x, x_{n}$

$$
d\left(T x, T x_{n}\right) \leq \lambda d(x, T x)^{\alpha} d\left(x_{n}, T x_{n}\right)^{1-\alpha}
$$

from which we deduce that $\lim _{n \rightarrow \infty} d\left(T x, T x_{n}\right)=0$.

Since $x \in X \backslash \operatorname{Fix}(T)$ was taken arbitrary, $T$ is constant on $X \backslash$ Fix $(T)$.

Theorem 2.3. Let $(X, d)$ be a metric space and $T: X \rightarrow X$ a Kannan-type contraction. Then, $T$ has a fixed point. 
FIXED POINT THEOREM FOR CRR-INTERPOLATIVE CONTRACTION ON NON-COMPLETE METRIC SPACES. 57

Proof. We shall consider the behaviour of $T$ on the subset $X \backslash$ Fix $(T)$.

For maps $T$ which are constant on $X \backslash \operatorname{Fix}(T)$, the existence of a fixed-point is easy to prove : Let $c$ in $X$ such that,

$$
\forall x \in X \backslash F i x(T), T x=c
$$

Suppose that $T(c) \neq c$, i.e. $c \in X \backslash F i x(T)$, then $T c=c$ which is a contradiction. Hence, $c$ is a fixed point of $T$.

For a map which is not constant on $X \backslash \operatorname{Fix}(T)$, we shall use the contraposition of proposition 2.2. It gives the following :

$$
m=\inf _{x \in X \backslash F i x(T)} d(x, T x)>0 .
$$

We will distinguish two cases :

First case : If $m$ is reached, i.e. there exists $\tilde{x} \in X$ such that $m=d(\tilde{x}, T \tilde{x})$. We apply (1.1) for $\tilde{x}$ and $T \tilde{x}$ and obtain:

$$
d\left(T \tilde{x}, T^{2} \tilde{x}\right) \leq \lambda d(\tilde{x}, T \tilde{x})^{\alpha} d\left(T \tilde{x}, T^{2} \tilde{x}\right)^{1-\alpha}
$$

and so

$$
d\left(T \tilde{x}, T^{2} \tilde{x}\right)^{\alpha} \leq \lambda d(\tilde{x}, T \tilde{x})^{\alpha}
$$

which gives :

$$
d\left(T \tilde{x}, T^{2} \tilde{x}\right) \leq \lambda^{\frac{1}{\alpha}} d(\tilde{x}, T \tilde{x})<d(\tilde{x}, T \tilde{x}),(0<\lambda<1)
$$

Let's set $y=T \tilde{x}$

$$
d(y, T y)=d\left(T \tilde{x}, T^{2} \tilde{x}\right)<m=\inf _{x \in X} d(x, T x) .
$$

Hence, $y \notin X \backslash \operatorname{Fix}(T)$. $y$ is a fixed point of $T$.

\section{Second case :}

If $m$ is not reached, there exists a sequence $\left(x_{n}\right)_{n} \subset X$ such that $\lim _{n \rightarrow \infty} d\left(x_{n}, T x_{n}\right)=m$. Applying (1.1) for $x_{n}$ and $T x_{n}$ gives :

$$
d\left(T x_{n}, T^{2} x_{n}\right) \leq \lambda d\left(x_{n}, T x_{n}\right)^{\alpha} d\left(T x_{n}, T^{2} x_{n}\right)^{1-\alpha}
$$

As in the first case :

$$
d\left(T x_{n}, T^{2} x_{n}\right) \leq \lambda^{\frac{1}{\alpha}} d\left(x_{n}, T x_{n}\right) .
$$

The right-side term tends to $\lambda^{\frac{1}{\alpha}} m<m$. If we take $n$ large enough to get $\lambda^{\frac{1}{\alpha}} d\left(x_{n}, T x_{n}\right)<m$, we obtain an element $y=T x_{n}$, such that :

$$
d(y, T y)<m=\inf _{x \in X} d(x, T x)
$$

Therefore, $y$ is a fixed point of $T$.

It is worth to notice that we used nowhere that $X$ is complete.

This concludes the proof for interpolative Kannan-type contractions. Now we shall give an analogous proof for CRR-type contractions. 
Proposition 2.4. Let $T$ be an interpolative $C R R$-type contraction on a metric space $(X, d)$. If $\inf _{x \in X \backslash F i x(T)} d(x, T x)=0$, then $T$ is constant on $X \backslash F i x(T)$.

Proof. Let $x \in X \backslash$ Fix $(T)$. According to the hypothesis,

$$
\exists\left(x_{n}\right)_{n \in \mathbb{N}} \subset X \backslash \operatorname{Fix}(T), \lim _{n \rightarrow \infty} d\left(x_{n}, T x_{n}\right)=0 .
$$

We use the inequality (1.2) for $x$ and $x_{n}$ to obtain

$$
d\left(T x, T x_{n}\right) \leq \lambda d\left(x, x_{n}\right)^{\alpha} d(x, T x)^{\beta} d\left(x_{n}, T x_{n}\right)^{\gamma}
$$

By the triangular inequality, we introduce $T x_{n}$ and $T x$ into the term $d\left(x, x_{n}\right)$, which gives:

$$
d\left(T x, T x_{n}\right) \leq \lambda\left(d(x, T x)+d\left(T x, T x_{n}\right)+d\left(x_{n}, T x_{n}\right)\right)^{\alpha} d(x, T x)^{\beta} d\left(x_{n}, T x_{n}\right)^{\gamma}
$$

Then we obtain the following inequality:

$$
\frac{d\left(T x, T x_{n}\right)}{\left(d(x, T x)+d\left(T x, T x_{n}\right)+d\left(x_{n}, T x_{n}\right)\right)^{\alpha}} \leq \lambda d(x, T x)^{\beta} d\left(x_{n}, T x_{n}\right)^{\gamma}
$$

Notice that the expression at the right-side of the inequality (2.2) converges to 0 .

Next, we prove that $\lim _{n \rightarrow \infty} d\left(T x, T x_{n}\right)=0$.

Suppose by contradiction that $d\left(T x, T x_{n}\right)$ does not converge to 0 . There exists a subsequence $\left(y_{n}\right)_{n}$ of $\left(x_{n}\right)_{n}$, and $\varepsilon>0$ for which:

$$
d\left(T y_{n}, T x\right)>\varepsilon, \forall n \in \mathbb{N}
$$

We will distinguish two cases :

First case : If the sequence $\left(d\left(T y_{n}, T x\right)\right)_{n \in \mathbb{N}}$ is bounded by $M \in \mathbb{R}^{+}$, we can extract from $\left(y_{n}\right)_{n}$ another subsequence $\left(z_{n}\right)_{n}$ such that $\left(d\left(T z_{n}, T x\right)\right)_{n \in \mathbb{N}}$ converges to $\ell \geq \varepsilon$, because $d\left(T y_{n}, T x\right) \subset$ $[\varepsilon, M]$ which is compact (Bolzano-Weierstrass).

For the subsequence $\left(z_{n}\right),(2.2)$ gives :

$$
\frac{d\left(T x, T z_{n}\right)}{\left(d(x, T x)+d\left(T x, T z_{n}\right)+d\left(z_{n}, T z_{n}\right)\right)^{\alpha}} \leq \lambda d(x, T x)^{\beta} d\left(z_{n}, T z_{n}\right)^{\gamma}
$$

by taking the limit when $n$ tends to $+\infty$, one obtains :

$$
\frac{\ell}{(d(x, T x)+\ell)^{\alpha}}=0
$$

Hence $\ell=0$ which contradicts $\ell \geq \varepsilon$.

Second case : If the sequence $\left.\left(d\left(T y_{n}, T x\right)\right)\right)_{n \in \mathbb{N}}$ is not bounded, we consider a subsequence $\left(z_{n}\right)_{n}$ of $\left(y_{n}\right)_{n}$ such that $\lim _{n \rightarrow \infty} d\left(T z_{n}, T x\right)=+\infty$. The formula (2.2) applied to the terms of $z_{n}$ gives :

$$
\frac{d\left(T x, T z_{n}\right)}{\left(d(x, T x)+d\left(T x, T z_{n}\right)+d\left(z_{n}, T z_{n}\right)\right)^{\alpha}} \leq \lambda d(x, T x)^{\beta} d\left(z_{n}, T z_{n}\right)^{\gamma} .
$$


FIXED POINT THEOREM FOR CRR-INTERPOLATIVE CONTRACTION ON NON-COMPLETE METRIC SPACES. 59

$\left(z_{n}\right)_{n}$ being a subsequence of $\left(x_{n}\right)_{n}$ we have $\lim _{n \rightarrow \infty} d\left(z_{n}, T z_{n}\right)=0$.

Hence, taking the limit in (2.3) gives us:

$$
\lim _{n \rightarrow \infty} \frac{d\left(T x, T z_{n}\right)}{\left(d(x, T x)+d\left(T x, T z_{n}\right)+d\left(z_{n}, T z_{n}\right)\right)^{\alpha}}=0
$$

In the other hand, notice that the following equivalence holds when $n$ tends to $\infty$ :

$$
\frac{d\left(T x, T z_{n}\right)}{\left(d(x, T x)+d\left(T x, T z_{n}\right)+d\left(z_{n}, T z_{n}\right)\right)^{\alpha}} \sim\left(d\left(T x, T z_{n}\right)\right)^{1-\alpha}
$$

Therefore,

$$
\lim _{n \rightarrow \infty} \frac{d\left(T x, T z_{n}\right)}{\left(d(x, T x)+d\left(T x, T z_{n}\right)+d\left(z_{n}, T z_{n}\right)\right)^{\alpha}}=+\infty
$$

which contradicts (2.4).

The two cases that we discussed lead to contradictions, we deduce that $\lim _{n \rightarrow \infty} T\left(x_{n}\right)=T(x)$. Since $x \in X$ was taken arbitrary, $T$ is constant on $X \backslash \operatorname{Fix}(T)$.

Theorem 2.5. Let $(X, d)$ be a metric space and $T: X \rightarrow X$ is interpolative CRR-type contraction. Then $T$ has a fixed point.

Proof. If $T$ is constant on $X \backslash \operatorname{Fix}(T)$, it is clear that $T$ has a fixed point (using exactly the same argument as for interpolative Kannan-type contractions). Now if $T$ is not constant on $X \backslash \operatorname{Fix}(T)$, by proposition $2.4, m=\inf _{x \in X \backslash \operatorname{Fix}(T)} d(x, T x)>0$.

We will distinguish the same two cases as for interpolative Kannan-type contractions :

First case :

If $m$ is reached. Let $\tilde{x} \in X$ such that $m=d(\tilde{x}, T \tilde{x})$. Applying theorem 2.5 for $\tilde{x}$ and $T \tilde{x}$ gives :

$$
d\left(T \tilde{x}, T^{2} \tilde{x}\right) \leq \lambda d(\tilde{x}, T \tilde{x})^{\alpha} d(\tilde{x}, T \tilde{x})^{\beta} d\left(T \tilde{x}, T^{2} \tilde{x}\right)^{\gamma}
$$

this implies :

$$
d\left(T \tilde{x}, T^{2} \tilde{x}\right)^{1-\gamma} \leq \lambda d(\tilde{x}, T \tilde{x})^{\alpha+\beta}
$$

Taking into consideration that $\alpha+\beta=1-\gamma$, one obtains :

$$
d\left(T \tilde{x}, T^{2} \tilde{x}\right) \leq \lambda^{\frac{1}{\alpha+\beta}} d(\tilde{x}, T \tilde{x})
$$

hence,

$$
d\left(T \tilde{x}, T^{2} \tilde{x}\right)<m
$$

We notice that for $y=T \tilde{x}$ we have:

$$
d(y, T y)<\inf _{x \in X \backslash F i x(T)} d(x, T x)
$$

Therefore, it must be the case that $y$ is a fixed point.

\section{Second case :}

If $m$ is not reached. There exists a sequence $\left(x_{n}\right) \subset X$ such that, $\lim _{n \rightarrow \infty} d\left(x_{n}, T x_{n}\right)=m$. Applying theorem 2.5 for $x_{n}$ and $T x_{n}$ gives :

$$
d\left(T x_{n}, T^{2} x_{n}\right) \leq \lambda d\left(x_{n}, T x_{n}\right)^{\alpha} d\left(x_{n}, T x_{n}\right)^{\beta} d\left(T x_{n}, T^{2} x_{n}\right)^{\gamma}
$$


hence,

$$
d\left(T x_{n}, T^{2} x_{n}\right) \leq \lambda^{\frac{1}{\alpha+\beta}} d\left(x_{n}, T x_{n}\right)(\alpha+\beta=1-\gamma) .
$$

The right-side term tends to $\lambda^{\frac{1}{\alpha+\beta}} m<m$. If we take $n$ large enough to get $\lambda^{\frac{1}{\alpha+\beta}} d\left(x_{n}, T x_{n}\right)<m$, we obtain an element $y=T x_{n}$, such that :

$$
d(y, T y)<m=\inf _{x \in X} d(x, T x) .
$$

$y$ is not in $X \backslash F i x(T)$, that is, $y$ is a fixed point.

The proof of the two theorems is now achieved, the reader may notice that the proofs of these two theorems are very similar, and are a little easier to establish for Kannan-type contractions. We choose to expose the previous theorems to show to the reader that the existence of a fixed point can be proved without considering Picard sequences. The next section is dedicated to their study.

\subsection{Behaviour of Picard sequences.}

Now, we give the proof of theorem 2.1, (page 3). As said in the introduction, unlike the theorems 1.1 and 1.2 this theorem gives the behaviour of Picard sequences for each point of the metric space.

Proof of theorem 2.1.

Case 1: $T$ is an interpolative Kannan-type contraction. Let $\alpha \in] 0,1[$ and $\lambda \in[0,1[$ such that

$$
\forall x, y \in X \backslash F i x(T), d(T x, T y) \leq \lambda d(x, T x)^{\alpha} d(y, T y)^{1-\alpha} .
$$

If $T$ is constant on $X \backslash \operatorname{Fix}(T)$, i.e., $T x=c, \forall x \in X \backslash \operatorname{Fix}(T)$. We proved in theorem 2.3 that $T c=c$. Hence, for arbitrary $x_{0}$ in $X, T^{2} x_{0}=T x_{0}$, and all Picard sequences are stationary. If $T$ is not constant on $X \backslash \operatorname{Fix}(T)$, we use the contraposition of the proposition 2.2 to obtain :

$$
m=\inf _{x \in X \backslash F i x(T)} d(x, T x)>0 .
$$

Let $x_{0} \in X$ and let $n \in \mathbb{N}$ such that

$$
\lambda^{\frac{n}{\alpha}} d\left(x_{0}, T x_{0}\right)<m .
$$

Suppose by contradiction that : $\forall k \in\{1, \ldots, n+1\}, T^{k} x_{0} \neq T^{k-1} x_{0}$. Then, for all $k \in$ $\{1, \ldots, n+1\}$, we obtain, by applying (2.5) with $x=T^{k-1} x_{0}$ and $y=T^{k} x_{0}$ :

and so

$$
d\left(T^{k} x_{0}, T^{k+1} x_{0}\right) \leq \lambda d\left(T^{k-1} x_{0}, T^{k} x_{0}\right)^{\alpha} d\left(T^{k} x_{0}, T^{k+1} x_{0}\right)^{1-\alpha}
$$

which gives :

$$
d\left(T^{k} x_{0}, T^{k+1} x_{0}\right)^{\alpha} \leq \lambda d\left(T^{k-1} x_{0}, T^{k} x_{0}\right)^{\alpha}
$$

$$
d\left(T^{k} x_{0}, T^{k+1} x_{0}\right) \leq \lambda^{\frac{1}{\alpha}} d\left(T^{k-1} x_{0}, T^{k} x_{0}\right)
$$

and by induction

$$
d\left(T^{n} x_{0}, T^{n+1} x_{0}\right) \leq \lambda^{\frac{n}{\alpha}} d\left(x_{0}, T x_{0}\right)<m
$$

we set $y=T^{n} x_{0}$ 
FIXED POINT THEOREM FOR CRR-INTERPOLATIVE CONTRACTION ON NON-COMPLETE METRIC SPACES. 61

$$
d(y, T y)<m=\inf _{x \in X \backslash F i x(T)} d(x, T x)
$$

Therefore, there exists an integer $1 \leq k \leq n+1$ such that $T^{k-1} x_{0}=T^{k} x_{0}$. And so, the sequence is stationary.

Case 2: $T$ is a CRR-type contraction. Let $\alpha, \beta, \gamma \in] 0,1[$, with $\alpha+\beta+\gamma=1$, and $\lambda \in[0,1[$ such that

$$
\forall x, y \in X \backslash F i x(T), d(T x, T y) \leq \lambda d(x, y)^{\alpha} d(x, T x)^{\beta} d(y, T y)^{\gamma}
$$

If $T$ is constant on $X \backslash \operatorname{Fix}(T)$, i.e. $T x=\mathcal{c}, \forall x \in X \backslash \operatorname{Fix}(T)$. We proved in theorem 2.5 that $T c=c$. Hence, for arbitrary $x_{0}$ in $X, T^{2} x_{0}=T x_{0}$, and all Picard sequences are stationary. If $T$ is not constant on $X \backslash F i x(T)$, we use the contraposition of the proposition 2.4 to obtain :

$$
m=\inf _{x \in X \backslash F i x(T)} d(x, T x)>0 .
$$

Let $x_{0} \in X$ and let $n \in \mathbb{N}$ such that

$$
\lambda^{\frac{n}{1-\gamma}} d\left(x_{0}, T x_{0}\right)<m
$$

Suppose by contradiction that $: \forall k \in\{1, \ldots, n+1\}, T^{k} x_{0} \neq T^{k-1} x_{0}$, then for all $k \in\{1, \ldots, n+1\}$, we obtain, by applying (2.6) with $x=T^{k-1} x_{0}$ and $y=T^{k} x_{0}$ :

$$
d\left(T^{k} x_{0}, T^{k+1} x_{0}\right) \leq \lambda d\left(T^{k-1} x_{0}, T^{k} x_{0}\right)^{\alpha} d\left(T^{k-1} x_{0}, T^{k} x_{0}\right)^{\beta} d\left(T^{k} x_{0}, T^{k+1} x_{0}\right)^{\gamma}
$$

and so

$$
d\left(T^{k} x_{0}, T^{k+1} x_{0}\right)^{1-\gamma} \leq \lambda d\left(T^{k-1} x_{0}, T^{k} x_{0}\right)^{\alpha+\beta}
$$

which gives (using the fact that $\alpha+\beta=1-\gamma$ ) :

$$
d\left(T^{k} x_{0}, T^{k+1} x_{0}\right) \leq \lambda^{\frac{1}{1-\gamma}} d\left(T^{k-1} x_{0}, T^{k} x_{0}\right)
$$

and by induction

$$
d\left(T^{n} x_{0}, T^{n+1} x_{0}\right) \leq \lambda^{\frac{n}{1-\gamma}} d\left(x_{0}, T x_{0}\right)<m
$$

we set $y=T^{n} x_{0}$

$$
d(y, T y)<m=\inf _{x \in X \backslash F i x(T)} d(x, T x)
$$

Therefore, there exists an integer $1 \leq k \leq n+1$ such that $T^{k-1} x_{0}=T^{k} x_{0}$. And so, the sequence is stationary.

Remark 2.6. This proof gives for each Picard sequence of initial $x_{0}$, an upper bound of the rank from which the sequence is stationary i.e., the lower rank $n \in N$ such that $T^{n}\left(x_{0}\right)=T^{n+1} x_{0}$. Namely, if we denote this rank by $N$, it satisfies:

$$
N \leq\left\lceil\frac{\alpha}{\ln (\lambda)} \ln \left(\frac{m}{d\left(x_{0}, T x_{0}\right)}\right)\right\rceil
$$


for interpolative Kannan-type contractions, and

$$
N \leq\left\lceil\frac{1-\gamma}{\ln (\lambda)} \ln \left(\frac{m}{d\left(x_{0}, T x_{0}\right)}\right)\right\rceil
$$

for interpolative CRR-type contractions, where $\lceil x\rceil$ is the integer value by excess of $x$.

Example 1. Let $X=\{0,1,2.5,10\}$ for the natural metric of $\mathbb{R}$ the map $\left(\begin{array}{llll}0 & 1 & 2.5 & 10 \\ 0 & 0 & 1 & 2.5\end{array}\right)$ is an interpolative CRR-type contraction for $\lambda=\frac{1}{2}, \alpha=\frac{3}{5}, \beta=\frac{1}{5}, \gamma=\frac{1}{5}$.

For $x_{0}=10$, we have

$$
\left\lceil\frac{1-\gamma}{\ln (\lambda)} \ln \left(\frac{m}{d\left(x_{0}, T x_{0}\right)}\right)\right\rceil=\left\lceil\frac{1-\frac{1}{5}}{\ln \left(\frac{1}{2}\right)} \ln \left(\frac{1}{7.5}\right)\right\rceil=3
$$

We deduce that the lower $N$ is less than 3 . In this case $N=3$ because $T^{2}(10)=1 \neq 0=T^{3}(10)$ and $T^{3}(10)=T^{4}(10)$. It is not true in general that $N=\frac{\alpha}{\ln (\lambda)} \ln \left(\frac{m}{d\left(x_{0}, T x_{0}\right)}\right)$. Indeed for the set $X=\{0,1,2.5,22.5\}$ and the map $\left(\begin{array}{cccc}0 & 1 & 2.5 & 22.5 \\ 0 & 0 & 1 & 2.5\end{array}\right)$, if we take $x_{0}=22.5, N=3$ but $\left\lceil\frac{1-\frac{1}{5}}{\ln \left(\frac{1}{2}\right)} \ln \left(\frac{1}{20}\right)\right\rceil=4$

\section{Finite CRR-type contractions}

In this section, we adopt the following notations :

$\Psi(X)$ the set of interpolative CRR-type contractions on X. We will simply note $\Psi$ when there is no ambiguity about the space we are considering.

We consider in this part a subset of $\Psi(X)$ which is of great interest. We define these sets as follows :

Definition 3.1. Let $X$ be a metric space.

An element $T$ of $\Psi(X)$ is said to be finite if the sequence $T^{n}$ is stationary, i.e.,

$$
\exists N \in \mathbb{N}, \forall n \geq N, \forall x \in X, T^{n} x=T^{n+1} x .
$$

We denote by $\Psi_{f}(X)$ the space of all finite elements of $\Psi(X)$

It is easy to see that for a finite set, $\Psi_{f}=\Psi$. But for infinite spaces we do not have an easy way to determine $\Psi_{f}$.

In the literature, there is no example of a space $X$ such that $\Psi(X) \neq \Psi_{f}(X)$. 
The first question that one can ask is whether or not there exists a space for which $\Psi(X)$ contains a non-finite element. We answer this question in the subsection 3.1.

In the subsection 3.2 we prove that if $X$ is bounded $\Psi_{f}(X)=\Psi(X)$. We also give in a special case, for $T \in \Psi_{f}(X)$ an upper bound to the rank from which $\left(T^{n}\right)_{n}$ is stationary. In the subsection 3.3 we prove that the boundedness of the space is necessary which gives us the main theorem of the section :

Theorem 3.1. Let $X$ be a metric space. The following assertions are equivalent:

- $X$ is bounded

- $\Psi_{f}(X)=\Psi(X)$

\subsection{Existence of infinite CRR-type contractions.}

In $\mathbb{N}$ endowed with the natural distance $|x-y|$, there exists for all $(\lambda, \alpha, \beta, \gamma)$ a non-stationary CRR-type contraction with these coefficients. Let consider the sequence :

$$
a_{0}=0, a_{1}=1, a_{n+1}>a_{n}+\left(\frac{a_{n}}{\lambda}\right)^{\frac{1}{\alpha+\min (\beta, \gamma)}}
$$

and define $T: \mathbb{N} \longrightarrow \mathbb{N}$ by:

$$
\begin{cases}\text { if } \quad x \in\left\{a_{n}, n \in \mathbb{N}\right\} & T a_{0}=0 \text { and } T a_{n}=a_{n-1} \\ \text { if } \quad x \notin\left\{a_{n}, n \in \mathbb{N}\right\} & T(x)=x .\end{cases}
$$

This sequence is strictly increasing, and :

$$
\forall i, j, 0<i<j, d\left(a_{j}, a_{j-1}\right)^{\alpha+\min (\gamma, \beta)}>\frac{a_{j}}{\lambda} .
$$

Since $\alpha+\min (\gamma, \beta) \leq \alpha+\beta<1$ and $d\left(a_{i}, T a_{i}\right) \geq 1$ we have the following :

$$
\lambda d\left(a_{j}, T a_{j}\right)^{\alpha+\beta} d\left(a_{i}, T a_{i}\right)^{\gamma}>a_{j}>d\left(T a_{j}, T a_{i}\right)
$$

and so,

$$
d\left(T a_{i}, T a_{j}\right) \leq \lambda d\left(a_{j}, a_{i}\right)^{\alpha} d\left(a_{j}, T a_{j}\right)^{\gamma} d\left(a_{i}, T a_{i}\right)^{\beta}
$$

taking $\alpha+\beta$ instead of $\alpha+\gamma$ gives the inequality where the role of $a_{j}$ and $a_{i}$ are reversed, we obtain :

$$
d\left(T a_{j}, T a_{i}\right) \leq \lambda d\left(a_{j}, a_{i}\right)^{\alpha} d\left(a_{j}, T a_{j}\right)^{\beta} d\left(a_{i}, T a_{i}\right)^{\gamma}
$$

$T$ is a CRR-type contraction and satisfy the following :

$$
\forall n \geq 1, T^{n} a_{n} \neq T^{n-1} a_{n} .
$$




\subsection{Finite Interpolative Kannan and CRR-type contractions for bounded metric spaces.}

Proposition 3.1. If $X$ is bounded by $M>0$, then $\Psi_{f}=\Psi$. In other terms, all CRR-type contractions defined on $X$ are finite.

Proof. Let $T$ be a CRR-type contraction on a metric space $X$. If $T$ is constant on $X \backslash$ Fix $(T)$, it is clear that $T \in \Psi_{f}$. Otherwise we consider : $m=\inf _{x \in X \backslash F i x(T)} d(x, T x)>0$, and let $x_{0} \in X$ and suppose that $T^{n+1}\left(x_{0}\right) \neq T^{n}\left(x_{0}\right)=x_{n}$.

$$
\begin{gathered}
\exists \lambda, \alpha, \beta, \gamma \in] 0,1[, \alpha+\beta+\gamma=1 \\
d\left(x_{n}, x_{n+1}\right)=d\left(T x_{n-1}, T x_{n}\right) \leq \lambda d\left(x_{n-1}, x_{n}\right)^{\alpha} d\left(x_{n-1}, x_{n}\right)^{\beta} d\left(x_{n}, x_{n+1}\right)^{\gamma}
\end{gathered}
$$

which gives

$$
d\left(x_{n}, x_{n+1}\right) \leq \lambda^{\frac{1}{\alpha+\beta}} d\left(x_{n}, x_{n-1}\right) \leq \lambda^{\frac{n}{\alpha+\beta}} d\left(x_{1}, x_{0}\right)
$$

in the other hand, it must be the case that :

$$
m \leq d\left(x_{n}, x_{n+1}\right) \leq \lambda^{\frac{n}{\alpha+\beta}} d\left(x_{1}, x_{0}\right) \leq \lambda^{\frac{n}{\alpha+\beta}} M .
$$

From the left-side and the right-side terms of the inequality we can deduce that :

$$
n \leq(\alpha+\beta) \frac{\log \left(\frac{m}{M}\right)}{\log (\lambda)}
$$

That is, $\left(T^{n}\right)_{n}$ is stationary, and the rank $N$ from which the sequence is stationary is bounded by $\left\lceil(\alpha+\beta) \frac{\log \left(\frac{m}{M}\right)}{\log (\lambda)}\right\rceil$.

\subsection{Existence of a non-finite CRR-type contraction on any non-bounded space.}

In this subsection, we prove that the boundedness of the space is a necessary condition, which means that there is equivalence between the boudedness of the space $X$ and the equality $\Psi_{f}=\Psi$.

Theorem 3.2. A metric space is bounded if and only if all CRR-type contraction defined on $X$ is of finite.

Proof. The direct implication is proved in Proposition 3.1.

For the other implication, we assume that $X$ is not bounded $(\delta(X)=\infty)$. Let $\lambda, \alpha, \beta, \gamma \in$ ] $0,1\left[, \alpha+\beta+\gamma=1\right.$. Let $\left(a_{n}\right)_{n}$ be a sequence of $X$ satisfying

$$
a_{0}, a_{1} \in X, d\left(a_{1}, a_{0}\right)>1, a_{n+1}: \inf _{1 \leq k \leq n} d\left(a_{n+1}, a_{k}\right) \geq\left(\frac{\delta\left(a_{k}, 0 \leq k \leq n\right)}{\lambda}\right)^{\frac{1}{\alpha+\min (\alpha, \beta)}}
$$

Let

$$
\left\{\begin{array}{ll}
T: & X \longrightarrow X \\
& x \longrightarrow x
\end{array} \quad \text { if } \quad x \notin\left\{a_{n}\right\} \text { or } x=a_{0}\right.
$$


Then, $T$ is a CRR-type contraction with coefficients $(\alpha, \beta, \gamma, \lambda)$. Indeed, $\forall i, j, 0<i<j$ :

$$
\begin{aligned}
& \lambda d\left(a_{i}, T a_{i}\right)^{\gamma} d\left(a_{j}, T a_{j}\right)^{\beta} d\left(a_{i}, a_{j}\right)^{\alpha} \geq \lambda d\left(a_{i}, T a_{i}\right)^{\gamma} d\left(a_{j}, T a_{j}\right)^{\min (\gamma, \beta)} d\left(a_{j}, a_{i}\right)^{\alpha} \\
& \geq \lambda \inf _{0 \leq k \leq j-1} d\left(a_{k}, a_{j}\right)^{\min (\gamma, \beta)+\alpha},\left(d\left(a_{i}, T a_{i}\right) \geq 1\right) \\
& \geq \delta\left(\left\{a_{k}, 0 \leq k \leq n\right\}\right) \\
& \geq d\left(T a_{i}, T a_{j}\right)
\end{aligned}
$$

To find the inequality where the roles of $a_{i}$ and $a_{j}$ are reversed we simply reverse the roles of $\gamma$ and $\beta$ in the previous calculation. $T$ satisfies :

$$
\forall n \geq 1, T^{n}\left(a_{n}\right) \neq T^{n-1}\left(a_{n}\right) .
$$

This ends the proof.

\section{Continuous interpolative CRR-type contractions}

In this last section, we study, for a metric space $X$, the set of continuous self-mappings in $\Psi(X)$ which we denote by $\Psi_{c}$. When the space contains only isolated points all self-mappings defined on it are continuous. But, for the metric spaces where the topology associated is not discrete there may exist non-continuous maps in $\Psi(X)$. We shall prove in this part a result in the specific case of connected spaces. Namely, we have the following :

Theorem 4.1. Let $X$ be a metric space, let $\Gamma(X)$ be the set of all continuous mappings that are constant on $X \backslash$ Fix $(T)$. If $X$ is connected, then,

$$
\Psi_{c}(X)=\Gamma(X)
$$

Proof. Let $X$ be a connected metric space. It is easy to see that

$$
\Gamma(X) \subset \Psi_{c}(X)
$$

Let $T \in \Phi_{c}(X) \backslash \Gamma(X)$, using the contraposition of Proposition 2.4, we obtain that $m=$ $\inf _{x \in X \backslash \operatorname{Fix}(T)} d(x, T x)>0$. In another hand, the function $D: x \in X \longrightarrow d(x, T x) \in \mathbb{R}^{+}$is continuous. $X$ being connected, $D(X)$ is connected in $\mathbb{R}^{+}$and so it is an interval. In addition, it has the following properties:

- $D(X) \subset\{0\} \cup[m,+\infty[$

- $0 \in D(X)$ because $T$ has a fixed point

- $D(X) \cap[m,+\infty[\neq \varnothing$

we conclude that $D(X)$ can't be connected. Thus there exists no map in $\in \Psi_{\mathcal{C}}(X) \backslash \Gamma(X)$ and $\Psi_{c}(X)=\Gamma(X)$. 


\section{References}

[1] R.P.Agarwal and E. Karapinar, Interpolative Rus-Reich-Ciric Type contractions Via Simulation Functions, An.St.Univ.Ovidius Constanta, Ser.Mat, Volume XXVII (2019) fasciola 3. Vol.27(3), 2019, 137-152.

[2] H. Aydi, E. Karapinar, and A. F. Hierro , $\omega$-Interpolative iri-Reich-Rus-Type Contractions, Mathematics, 2019, 7,57. https://www.mdpi.com/journal/mathematics

[3] Y.U.Gaba, E.Karapinar, A new approach to the interpolative Contractions, Axioms 2019, 8, 110.

[4] E. Karapnar, Revisiting the Kannan Type Contractions via Interpolation, Advances in the Theory of Nonlinear Analysis and its Applications 2 (2018) No. 2, 8587.

[5] E.Karapinar, R.Agarwal, H.Aydi, Interpolaive Reich-Rus-Ciric Type Contractions on Partial Metric spaces, Mathematics 2018, 6, 256. https://doi.org/10.3390/math6110256.

[6] E.Karapinar, O.Alqahtani, H.Aydi, On interpoaltive Hardy-Rogers type Contractions, Symmetry 2019, 11(1),8; https: / / doi.org/10.3390/math7010084.

[7] M. Noorwaly, Journal of Mathematical Analysis.

ISSN: 2217-3412, URL: www.ilirias.com/jma

Volume 9 Issue 6 (2018), Pages 92-94. 